\title{
Merozoites of Theileria Orientalis Buffeli Reduce Parasitosis Following Challenge by Ticks Infested with T.Orientalis Ikeda
}

David Emery ( $\nabla$ david.emery@sydney.edu.au )

Univertsity of Sydney https://orcid.org/0000-0003-2722-6854

\section{Susan de Burgh}

U1 University

Therese Hoang Hieu Hanh Dinh

Sydney University

\section{Peter Rolls}

Tick Fever Centre

\section{Phillip Carter}

Tick fever Centre

\section{Research}

Keywords: Theileria, T.orientalis buffeli, T.orientalis ikeda, carrier state, Haemaphysalis, cattle DOI: https://doi.org/10.21203/rs.3.rs-92267/v1

License: (c) (1) This work is licensed under a Creative Commons Attribution 4.0 International License. Read Full License 


\section{Abstract}

Background: An investigation aimed to confirm whether immunisation with the "benign" buffeli genotype of Theileria orientalis could reduce the parasitosis of the virulent ikeda genotype.

Methods: Calves were inoculated intravenously or subcutaneously with bovine blood containing merozoites of T.orientalis buffeli. When recipients became positive, they and control animals were challenged with unfed nymphs of Haemaphysalis longicornis ticks infested as larvae with T.orientalis ikeda.

Results: All calves became positive for the challenge within 12 days after tick application. In the immunised calves, the first wave of parasitosis with T.orientalis ikeda from 4-6 weeks was reduced significantly by $>80 \%$ before the infestation declined into the carrier state by 9 weeks.

Conclusions: The results confirm the field experience with theileriosis in endemic zones where the carrier state appears to prevent clinical disease despite repeated, seasonal tick infestations with virulent genotypes of the parasite. This method offers a means to reduce the severity of the first wave of theilerial parasitosis after tick challenge and recover associated production losses.

\section{Background}

The intracellular protozoal parasite Theileria orientalis has rapidly spread across South-eastern Australia, substantially impacting local cattle industries since 2006. Given that Theileria buffeli, causing "benign theileriosis" had been present in Queensland since 1912 (Stewart et al.,1992; 1996), the new syndrome was termed "Bovine Anaemia caused by the T heileria o rientalis group" (BATOG; Eamens et al., 2013a). Molecular techniques have since enabled discrimination of the T.orientalis genotypes and identified genotypes "ikeda" and "chitose" as those causing clinical disease (Kamau et al., 2011; Eamens et al., 2013a,b; Bogema et al., 2015). The basis for the current classification uses the major piroplasm surface protein (MPSP), which is expressed in the intraerythrocytic stage of $T$. orientalis and conserved to some extent among different geographic isolates. It has been widely used for molecular epidemiological studies of T. orientalis in Japan (Ota et al., Sivakumar et al., 2012), Korea (Park et al., 2017), Kenya, and Australia (Izzo et al., 2010; Kamau et al., 2011; Bogema et al., 2015). Currently, 11 genotypes of $T$. orientalis (type 1 or chitose, type 2 or ikeda, type 3 or buffeli, types $4-8$, and N1-N3) had been identified based on MPSP gene sequences (Sivakumar et al., 2014; Bogema et al., 2015). Of these genotypes, 1 and 2 cause the majority of clinical disease in cattle, with the phylogenetic cluster chitose A mostly associated with the ikeda genotype in clinical cases (Jenkins et al., 2015). On farms impacted by the parasite, NSW-DPI have estimated an average cost of $\$ 59 \mathrm{~K}$ for dairy producers and $\$ 11.6 \mathrm{~K}$ for beef producers, which equates to AUD $\$ 131 /$ head for dairy cattle and AUD $\$ 67 /$ head for beef cattle (Bailey 2012). Similar losses have been reported from Victoria (Perrera et al., 2014). The total cost of theilerosis in Australia was estimated at around $\$ 20 \mathrm{~m}$ pa nationally (Lane et al., 2015). Recovered cattle remain carriers of theilerial genotypes (Eamens et al., 2013b; Hammer et al., 2016) and several studies have 
indicated that the carrier state in dairy cattle did not compromise subsequent productivity (Perrera et al., 2014; Lawrence et al., 2019).

Interestingly, inoculation of naïve cattle with blood stages of T.orientalis genotypes does not cause clinical disease (Hammer et al., 2016; Gibson, 2017), but a carrier state is established and parasitosis is detectable by PCR in blood for greater than 30 months (Hammer et al., 2016; unpublished). Irrespective of the theilerial genotype(s) present, the carrier state arising after natural tick-borne infection appears to prevent recurrence of clinical disease following seasonal tick challenge in endemic regions (Izzo etal, 2012; Kamau et al., 2011; Eamens et al., 2013b). This has been witnessed by the progression of the epidemic curve through eastern and southern Australia and New Zealand, with mainly newborn calves and introduced cattle remaining susceptible to clinical disease in endemic zones (Bailey, 2012; Jenkins et al., 2015). Similarly, the widespread presence of T.orientalis buffeli carrier cattle in Queensland has been attributed to the low prevalence of virulent genotypes and/or clinical theileriosis in that state (de Vos, 2011). In endemic regions, infestations of T.orientalis buffeli in susceptible cattle were clearly outpaced by virulent theilerial genotypes ikeda and chitose in both Gloucester and Dorrigo (Jenkins et al., 2015; DE, unpublished), negating any opportunity to induce protection. Leaving vector competence aside, some type and level of immunity exists in carrier cattle which resembles "premunity" (Neal et al., 1969), interfering with the severity of subsequent challenge infections. This is witnessed in the long histories of early "vaccinations" against leishmania, malaria, East Coast fever, babesiosis and poultry coccidiosis ("precocious strains") (see McAllister, 2014).

Consistent with the circumstantial and historical evidence for the operation of "premunity" against repeated seasonal tick infestation with T.orientalis, this study examined whether immunisation with blood stages (merozoites) of $T$. orientalis buffeli could reduce the severity of subsequent T.orientalis ikeda infestation. This study also enabled an opportunity to determine whether $H$. longicornis nymphal ticks infected as larvae on an infected calf could transmit that infection as nymphs, to an uninfected second host, and then retain that ability to re-infect a third host as adult ticks.

\section{Materials And Methods}

\subsection{Theilerial stabilates.}

T.orientalis ikeda. Blood was collected from the jugular vein of a donor cow into lithium heparin blood collection tubes at Camden NSW, $28 \mathrm{ml}$ of which was subsequently inoculated into a splenectomised calf at the Tick Fever Centre, Wacol, Qld. Approximately 1 month after inoculation, a stabilate (designated J36) was produced using $100 \mathrm{ml}$ of blood collected into sodium heparin mixed with an equal volume of $20 \%$ polyvinylpyrrolidone (PVP 40,000; $\mathrm{pH} 7.2$ ) cryoprotectant solution. This blood mixture was decanted into each of $5 \mathrm{ml}$ cryotubes The cryotubes were then placed in the vapour phase of liquid nitrogen for 15 minutes before the stabilate was lowered into the liquid nitrogen for storage. The stabilate contained $2 \times$ $10^{8}$ merozoites per ml. For infection of ticks, unfed adult $H$. longicornis ticks which had previously fed on a splenectomised calf that had been inoculated with a thawed $5 \mathrm{ml}$ cryotube of $\mathrm{J} 36$ stabilate, were 
placed on a subsequent splenectomised calf (3604) and transmitted T. orientalis ikeda. Additional clean larval ticks were then fed on this $T$. orientalis Ikeda-infested calf to themselves become infected. The engorged larval ticks were then collected and allowed to moult to nymphs in the incubator before allpication.

T.orientalis buffeli. A blood stabilate (J46) was similarly prepared from a cow at Bairnsdale, Victoria and stored in liquid nitrogen at the TFC. To prepare the blood inoculum for this trial, splenectomised calf 3584 was inoculated with a thawed $5 \mathrm{ml}$ cryotube of stabilate intravenously and blood collected some months later.

\subsection{Calves and infection.}

The trials in this study conformed with requirements for animal health and well-being under the University of Sydney Animal Ethics Committee (AEC) permit 2018/1328.

Fifteen Holstein (neutered male) calves aged 3-4 months were purchased from Leppington Pastoral Company (LPC, Cobbity, NSW) and housed on pasture at The University of Sydney's Pye Farm, Greendale NSW. Calves were weighed and bled to confirm their negative theilerial status by AusDiagnostics ${ }^{\circledR}$ Multiplex-Tandem PCR (MT-PCR) before being randomly assigned to 3 treatment groups, each of 5 animals. Group 1 calves were premedicated with $100 \mathrm{mg}$ of the antihistamine chlorpheniramine maleate (Histamil ${ }^{\circledR}$ ) and $15 \mathrm{mg}$ dexamethasone ( Dexapent ${ }^{\circledR}$ ) by intramuscular injection, to lower the risk of any reaction from blood incompatibility. After $30 \mathrm{~min}$, each calf was given a $5 \mathrm{ml}$ intravenous (IV) infusion of fresh infected blood obtained from an infected steer at the TTFC, (steer 3584, infected with T.orientalis buffeli blood stabilate). Previous screening by PCR indicated a specific infection containing $1.12 \times 10^{5}$ gene copies of T.orientalis buffeli per ul blood. The inoculum contained $1.3 \times 10^{6}$ merozoites of T.orientalis buffeli per $\mathrm{ml}$. All calves became PCR positive within 28d. Group 2 calves were to be infested with $100 \mathrm{H}$.bancroftinymphs (infected with T.orientalis buffeli as larvae) from TFC under patches, but these ticks died on steer 3584. Consequently, H.longicornis larvae were fed on steer 3584, moulted, and approx. 200 unfed nymphs were applied to each calf in Group 2 under backline calico patches as described by Marendy et al. (2019). Ticks were collected and removed after 6 days when they were engorged. When these 5 calves failed to become PCR positive after 5 weeks, $5 \mathrm{ml}$ of fresh infected blood containing $9 \times 10^{8}$ T.orientalis buffeli merozoites per $\mathrm{mL}$ (from steer 3584) was inoculated subcutaneously (SC) into each calf. All these 5 calves were PCR positive for T.orientalis buffeli within $21 d$.

Thirteen weeks after Group 1 calves and 28 days after Group 2 calves were inoculated with T.orientalis buffeli blood, approximately 200 unfed H.longicornis nymphs, previously infected with T.orientalis ikeda as larvae at TFC, were placed under backline patches on each of the 15 calves (including the uninoculated group 3 controls). The ticks were collected from each calf after 6 days, counted and pooled, before posting to the Biosecurity Sciences Laboratory (BSL, Brisbane, Qld), to moult to adults; 10 were also placed into $100 \%$ ethanol for PCR. When moulted and their exoskeletons had hardened (Marendy et al.,2019), around 50 of these unfed adult H.longicornis were applied to each of 3 naïve calves to 
determine if the original T.orientalis ikeda infection persisted through the nymphal stage on the uninfected calves and survived through the moult to adult ticks.

All calves were monitored visually daily, with $5 \mathrm{ml}$ blood collected weekly into EDTA vacuum tubes (Vacuette, Griener Bio-one) from day 14 post-inoculation of buffeli blood and from days 14-62 after $T$. orientalis ikeda infected, tick challenge.

\subsection{Sample analyses.}

PCV was measured by centrifuging blood in micro-haematocrit tubes (Beckman Coulter centrifuge). Diagnostic PCR was performed by DNA extraction from 200 ul blood samples using the MagMax ${ }^{\text {TM }}$ CORE Nucleic Acid Purification kit (ThermoFisher Scientific Inc.). A KingFisher Duo ${ }^{\text {TM }}$ Prime Magnetic Particle Processor completed DNA isolation.

Theilerial PCR runs were conducted with 11 eluted DNA samples, one indicator sample, MasterMixes (Theileria), oil, water, and step 2 strips loaded separately into the AusDiagnostics ${ }^{\circledR}$ Easy-Plex ${ }^{\text {mm }}$ Processor, which provided sequences for theilerial genotypes ikeda, buffeli, chitose, and type 5. Both automatic assay set-up and Step 1 PCR were accomplished in this machine. Samples were then transferred into the Bio-rad CFX96 analyser to perform the Step 2 PCR. Real-time PCR Ct-values were collected for data analysis. Relative gene copy numbers per ul of blood (GP/ul) were calculated for both pan-theileria and theilerial genotypes from spiked standards in the assay.

\subsection{Statistical analysis.}

Statistical analysis was performed using GenStat ${ }^{\mathrm{T}}$. Raw data were $\log _{\mathrm{e}}$ transformed and two linear mixed models for PCV and ikeda gene copies per $\mu \mathrm{L}$ were developed for each study as appropriate. The fixed effects were Day, Treatment and the interaction between Day and Treatment. The random effect was Animal ID. P-values were calculated for each fixed effect in each model to determine significance. For significant fixed effects, the differences in the predicted means for each factor level were compared to the Least Significant Differences (LSDs) at significance level 0.05 to determine whether pairwise comparisons were significant. If the interaction fixed effect was significant, no further pairwise comparisons were determined for the other fixed effects.

Predicted means for PCV and GC/ul for theilerial genotypes within treatment groups were presented with standard error (SE) bars.

\section{Results}

\subsection{Immunisation with T.orientalis buffeli.}

Both intravenous (IV) and subcutaneous (SC) inoculation of bovine blood infected with T.orientalis buffeli produced parasitosis detectable by PCR within 4 weeks of inoculation. Over the 13 weeks before challenge, the parasitosis in Group 1 (IV) reached a mean peak of 5,063 GC/ul of T.orientalis buffeli by 5 
weeks, decreasing and stabilising 9-13 weeks after initial inoculation between means of 1292 and 876 $\mathrm{GC} / \mathrm{ul}$ (Table 1). By comparison, at the time of challenge, 4 weeks after inoculation SC, Group 2 calves had a mean parasitosis of $1547 \mathrm{GC} / \mathrm{ul}$ (Table 1). Following the T. orientalis Ikeda infected, tick challenge, the parasitosis with T.orientalis buffeli remained relatively steady in both Groups 1 and 2 over the next 60 days at $<2500 \mathrm{GC} / \mathrm{ul}$.

Table 1

Parasitosis of T.orientalis buffeli from calves in Groups $1 \& 2$ after inoculation of infested blood.

\begin{tabular}{|c|c|c|c|c|c|c|c|c|c|}
\hline \multirow{2}{*}{$\begin{array}{l}\text { Group } \\
\text { treatment }\end{array}$} & \multicolumn{9}{|c|}{ Days after tick infestation } \\
\hline & $-62^{\star}$ & -56 & -28 & 0 & 12 & 18 & 35 & 47 & 62 \\
\hline \multirow[t]{2}{*}{ IV ( Gp1) } & $\begin{array}{l}9144 \\
+/-85\end{array}$ & $\begin{array}{l}5063 \\
+/-72\end{array}$ & $\begin{array}{l}1292 \\
+/-21\end{array}$ & $876+/-$ & $\begin{array}{l}133 \\
+/-\end{array}$ & $\begin{array}{l}3+/- \\
02\end{array}$ & $\begin{array}{l}3+/- \\
02\end{array}$ & $\begin{array}{l}3+/- \\
0.3\end{array}$ & $\begin{array}{l}2+/- \\
02\end{array}$ \\
\hline & & & & 0.67 & 0.65 & & & & \\
\hline \multirow[t]{2}{*}{$\mathrm{SC}(\mathrm{Gp} 2)$} & na & na & na & $\begin{array}{l}1547 \\
+/-0.7\end{array}$ & $\begin{array}{l}157 \\
+/-\end{array}$ & $\begin{array}{l}20+/- \\
0.65\end{array}$ & $\begin{array}{l}24+/- \\
0.6\end{array}$ & $\begin{array}{l}20+/- \\
0.4\end{array}$ & $\begin{array}{l}12+/- \\
0.4\end{array}$ \\
\hline & & & & & 0.65 & & & & \\
\hline \multicolumn{10}{|c|}{ Results are expressed as mean GC/ul blood +/- SE. } \\
\hline \multicolumn{10}{|c|}{ * 4 weeks after inoculation of blood IV; na = not applicable } \\
\hline
\end{tabular}

Following the initial inoculations of blood into Group 1, it was revealed that 2 calves in the control Group 3 exhibited pre-existing low parasitoses with T.orientalis ikeda of $<500 \mathrm{GC} / \mathrm{ul}$, presumably from infections acquired in utero or postnatally. This meant that these 2 calves were excluded from the control group (3 calves) for statistical analysis, but were included for analysis as a separate group (of 2 calves) after tick infestation.

\subsection{Effects of T.orientalis buffeli on T.orientalis ikeda after tick challenge.}

All 15 calves in the 3 groups became positive for T.orientalis ikeda within 12 days after infestation (DAl) from the application of the 200 infected H.longicornis nymphs. The parasitosis in the control groups followed a typical pattern in peaking around 5 weeks (39 DAl) after infestation at a mean $69734 \mathrm{GC} / \mathrm{ul}$ before declining to $<2000 \mathrm{GC} / \mathrm{ul}$ blood by $62 \mathrm{DAl}$ (Fig. 1, Table 2). The parasitosis in the 3 treatment groups were significantly reduced between 30 and $85 \%$ on sampled days during the first wave of parasitaemia from 25-39 DAI (Fig. 1, Table 2). In parallel, the PCV in the control group decreased by 16$20 \%$ after infestation to a mean of $25 \%$ by $39 \mathrm{DAl}$, significantly reduced compared to the SC immunised group 2 (Table 3) and remained significantly lower than group 2 up to 62 DAI (Table 3). The PCV of the IVimmunised group 4 was only significantly higher than control group 1 on 32DAI (Table 3). 
Table 2

Parasitosis of T.orientalis ikeda in treatment groups after challenge with infested H.longicornis.

\begin{tabular}{|c|c|c|c|c|c|c|c|}
\hline Groups & Day 12 & Day 18 & Day 25 & Day 32 & Day 39 & Day 47 & $\begin{array}{l}\text { Day } \\
62\end{array}$ \\
\hline $\begin{array}{l}\text { Control Gp3 } \\
\text { (3) }\end{array}$ & $\begin{array}{l}189 \\
(97)^{\text {ab }}\end{array}$ & $\begin{array}{l}9180 \\
(4690)^{a}\end{array}$ & $\begin{array}{l}33134 \\
(18585)^{a}\end{array}$ & $\begin{array}{l}30303 \\
(15500)^{a}\end{array}$ & $\begin{array}{l}68734 \\
(8969)^{a}\end{array}$ & $\begin{array}{l}18251 \\
(9150)^{a}\end{array}$ & $\begin{array}{l}84 \\
(588)^{a}\end{array}$ \\
\hline IV (5) & $\begin{array}{l}94 \\
(37)^{a}\end{array}$ & $\begin{array}{l}2800 \\
\left(1110^{a}\right.\end{array}$ & $1124(445)^{\mathrm{c}}$ & $\begin{array}{l}3446 \\
(1360)^{b}\end{array}$ & $\begin{array}{l}9740 \\
(6414) \\
\text { b }\end{array}$ & $\begin{array}{l}10027 \\
(6253) \\
a\end{array}$ & $\begin{array}{l}2855 \\
(1588) \\
b\end{array}$ \\
\hline $\begin{array}{l}\text { *Controls + } \\
\text { ve }(2)\end{array}$ & $\begin{array}{l}1181 \\
(835)^{b}\end{array}$ & $\begin{array}{l}9063 \\
(6409)^{a}\end{array}$ & $\begin{array}{l}6741 \\
(4767)^{a b}\end{array}$ & $\begin{array}{l}11464 \\
(8107)^{a b}\end{array}$ & $\begin{array}{l}6815 \\
(4374) \\
\text { b }\end{array}$ & $\begin{array}{l}810 \\
(632)^{b}\end{array}$ & $\begin{array}{l}422 \\
(228) \\
a\end{array}$ \\
\hline SC (5) & $\begin{array}{l}488 \\
(193)^{b}\end{array}$ & $\begin{array}{l}9200 \\
(3640)^{a}\end{array}$ & $\begin{array}{l}4020 \\
(1590)^{b}\end{array}$ & $\begin{array}{l}3648 \\
(1440)^{b}\end{array}$ & $\begin{array}{l}9154 \\
(9180) \\
\text { b }\end{array}$ & $\begin{array}{l}3637 \\
(4224) \\
\text { ab }\end{array}$ & $\begin{array}{l}432 \\
(331) \\
a\end{array}$ \\
\hline \multicolumn{8}{|c|}{$\begin{array}{l}\text { Results are expressed as mean GC/ul blood +/-SE; * the } 2 \text { calves in Gp3 animals found positive for } \\
\text { T.orientalis prior to tick challenge }\end{array}$} \\
\hline
\end{tabular}

Table 3

Changes in haematocrit in treatment groups after challenge with infested H.longicornis.

\begin{tabular}{|llllllll|}
\hline Groups & Day 12 & Day 18 & Day 25 & Day 32 & Day 39 & Day 47 & Day 62 \\
\hline Control (3) & $32^{\mathrm{a}}$ & $38.3^{\mathrm{a}}$ & $31.7^{\mathrm{a}}$ & $25^{\mathrm{a}}$ & $26.7^{\mathrm{a}}$ & $27^{\mathrm{a}}$ & $27.3^{\mathrm{a}}$ \\
\hline IV (5) & $27.8^{\mathrm{a}}$ & $30.8^{\mathrm{a}}$ & $32^{\mathrm{a}}$ & $29.8^{\mathrm{b}}$ & $30.2^{\mathrm{ab}}$ & $30.4^{\mathrm{ab}}$ & $28.2^{\mathrm{ab}}$ \\
\hline$*^{*}$ Controls + ve (2) & $35^{\mathrm{a}}$ & $33.5^{\mathrm{a}}$ & $36^{\mathrm{a}}$ & $27.5^{\mathrm{ab}}$ & $29.5^{\mathrm{ab}}$ & $32^{\mathrm{b}}$ & $31.5^{\mathrm{bc}}$ \\
\hline SC (5) & $28.8^{3}$ & $33.6^{\mathrm{a}}$ & $31.6^{\mathrm{a}}$ & $28.6^{\mathrm{ab}}$ & $31.6^{\mathrm{b}}$ & $33.2^{\mathrm{b}}$ & $31.8^{\mathrm{c}}$ \\
\hline Results are expressed as \% PCV. & & & & & \\
\hline
\end{tabular}

\subsection{Persistence of theilerial infestation between larval and adult stages of H.longicornis..}

Moulted adult ticks derived from the application of T.orientalis ikeda- infested nymphs of H.longicornis onto 15 uninfected calves (as a second host) were reapplied to 3 naive calves as third hosts. Blood 
samples at 18 DAl were positive for T.orientalis ikeda with 920,3150 and 10,280 GC/ul in the 3 calves, indicating that the ticks retained infectivity through 2 moults.

\section{Discussion}

Both intravenous (IV) and subcutaneous (SC) inoculation of bovine blood infected with T.orientalis buffeli produced parasitosis detectable by PCR within 4 weeks, consistent with previous reports (Hammer et al., 2016; Gibson, 2017). In each case the parasitosis appeared to peak around 5-8 weeks before stabilising at around 2000-10000 GC/ul. It was also noted when H.bancroftiticks failed to adequately feed and died, that H.longicornis nymphs failed to transmit T.orientalis buffeli to infect naive calves. These results were consistent with previous vector studies indicating that $H$.bancrofti and H.humerosa were likely vectors for T.orientalis buffeli in Queensland (Stewart et al., 1987a,b). H. Iongicornis occurs in the coastal areas of Victoria and New South Wales and extends northwards as far as Gympie in Queensland but is absent from large areas of Northern Australia where Theileria sp (T.orientalis buffeli) is present (Reik, 1982).

Prior inoculation of T.orientalis buffeli-infected blood containing between $6.5 \times 10^{6} \mathrm{GC} / \mu \mathrm{L}$ (iv) or $4 \times 10^{9}$ $\mathrm{GC} / \mu \mathrm{L}$ (sc) and allowed to "consolidate/incubate" for 13 and 4 weeks, respectively, before T.orientalis ikeda- infected ticks were applied, significantly reduced the initial parasitosis of T.orientalis ikeda over the next 50 days. It was also observed that 2 calves already serendipitously parasitised with $<500 \mathrm{GC} / \mathrm{ul}$ T.orientalis ikeda on arrival at 4 months of age, presumably in utero or post-natally, were also significantly "protected" from the first wave of parasitosis following tick challenge. These outcomes consolidated several older theilerial reports from Japan and Korea (Baek et al., 1982; Minami et al., 1981; Onuma et al., 1997) with T.orientalis sergenti, which has been confirmed as T.orientalis (Stewart et al., 1996). A cryopreserved vaccine containing $2 \times 10^{8}$ red blood cells containing T.orientalis [sergenti] per dose "had an inhibitory effect on the clinical manifestation of theileriosis" with a need for proliferation of the inoculum (Ishihara, 1962) but this was not developed further. Production of an attenuated whole blood vaccine against $T$. orientalis [sergenti] occurred in Korea but outcomes were not reported and challenge appeared to use blood stabilate (Baek et al.,1992). Later, sonicated T. orientalis [sergenti] merozoites produced significant reductions in parasitosis after 3 months, among recipients receiving 2 doses of $100 \mathrm{mg}$ in complete Freund's adjuvant subcutaneously, 1 month apart, and subjected to field infestation from 2- 5 months after initial vaccination (Baek et al., 1992). Unfortunately, the trial was terminated after 5 months as all controls and $20 \%(4 / 20)$ of vaccinates required treatment with diminazene (Berenil $\circledast$ ) for anaemia (Baek et al., 1992). A recombinant MPSP vaccine for T.orientalis [sergenti] utilised 3 vaccinations at 3 week intervals, producing an antibody response but no protection against challenge (Park et al, 1999).

Overall, these reports and the current trial support the premise that prior infestation with either T.orientalis ikeda or buffeli merozoites, effectively generates a state of "premunity" which mitigates the severity of a subsequent tick challenge with T.orientalis ikeda (Neal et al., 1969; Stewart et al., 1992). This situation reflects field experience where recovered cattle may harbour multiple theilerial genotypes in the carrier 
state. It should be noted that inoculation of merozoites of T.orientalis ikeda into susceptible cattle does not appear to cause the clinical episodes of theileriosis that follows tick infestation (Hammer et al., 2016; this study). Such a method of "immunisation" may be a feasible means of control against ticks carrying the virulent theilerial genotypes.

These results are also consistent with the susceptibility of introduced cattle and calves in endemic regions with multiple theilerial genotypes. As noted with the kinetics and age-related infections of calves and introduced stock (Jenkins et al., 2015; Swilks et al., 2017) and in naïve animals at Dorrigo, T.orientalis buffeli infestations are the slowest to develop (Emery et al., unpublished). Results indicated that infections with the virulent genotypes (ikeda and chitose) occurred earlier with maximal parasitosis around 5- 6 weeks before declining, whereas parasitosis with $T$. orientalis buffeli was detectable in an increasing proportion of naïve animals up to 3-4 months before stabilising (Jenkins et al., 2015; Emery et al., unpublished). All animals remained carriers of all four genotypes in the AusDiagnostics $®$ PCR kit for at least 6 months and $>80 \%$ of ticks sampled from Dorrigo and Stroud contained DNA from the four theilerial genotypes (ikeda, chitose, Type 5 and buffeli). The earlier appearance of the virulent genotypes negates any opportunity for endemic T.orientalis buffeli to generate any host-protective capability before challenge with virulent genotypes.

This study is the first confirmation of speculations that the carrier state persisting in cattle which have recovered from the initial parasitosis with virulent theilerial genotypes, establishes some type of "protection" to subsequent, seasonal tick challenge. As recommended by de Vos (2011), dose-response studies with single or mixed genotypes are required to establish vaccination potential. Field trials in endemic regions with high levels of tick infestation harbouring multiple theilerial genotypes are also vital to determine the robustness of the method, and whether there is any further "protection" conferred to parasitosis induced by subsequent tick challenges. Therefore, this outcome is being repeated in a field trial providing an intense natural challenge and to test homologous protection using T.orientalis ikeda blood. It may be that prior immunisation with merozoites from any genotype(s) of T.orientalis may reduce the severity of parasitosis following challenge with virulent genotypes as appears to be the case in numerous regions of infested with T.orientalis.

In an additional observation on the vector competence of H.longicornis for T.orientalis ikeda, unfed nymphs infected as larvae effectively transmitted the parasite to naïve second host calves and after collection and moulting to adult stages, could readily transmit T.orientalis ikeda to a further group of naïve calves. This indicated that the tick retained an initial infection acquired in the larval stage, through two moults to the adult stage and did not require any "boosting" from the second host. In the field, this implies that the nymphal stage could engorge on companion or wildlife second hosts and still remain infective for cattle as their third host.

\section{Conclusions}


Calves inoculated SC or IV with blood infested with merozoites of T.orientalis buffeli became positive for the parasite by PCR around 28 days after infestation (DAl) and did not develop clinical signs over the following 9 weeks. Following the application of 200 H.longicornis nymphs harbouring T.orientalis ikeda, the first peak of parasitosis occurring from 25-47 DAl was reduced by around $80 \%$ in immunised calves compared to control animals. These results are consistent with the lack of clinical theileriosis in carrier cattle in endemic regions and strongly suggest that host regulation maintains theilerial populations of the virulent genotypes below levels causing clinical disease, despite repeated seasonal tick challenges. The results also imply that prior vaccination with blood forms of the parasite could reduce deaths and production losses incurred from the first wave of parasitosis following tick infestation with virulent theilerial genotypes ikeda and chitose A.

\section{Declarations}

Competing interests. The authors declare that they have no competing interests

\section{Acknowledgements}

This project was funded by Meat \& Livestock Australia Donor Company (MLADC), Project P.PSH.0832. The excellent assistance of Mr Ralph Sutchbury and Ms Cath Covaicin at Queensland's Department of Agriculture and Fisheries Biosecurity Sciences Laboratory (BSL), Brisbane in moulting and providing ticks is greatly appreciated. We are extremely grateful for the statistical support from Dr Evelyn Hall, technical support from Ms. Maira Meggiolaro and the field assistance by the staff at the University of Sydney Pye Farm.

\section{References}

1. Baek BK, Kim BS, Choi .H, et al. Immunogenicity and protective efficacy of solubilized merozoiteenriched Theileria sergenti immunogens. II: Protection against natural exposure under field conditions. Kor J. Parasitol, 1992; 30: 201-208. http://dx.doi.org/10.3347/kjp.1992.30.3.201

2. Bailey G. Theileria in cattle- a farmers guide to a "new" disease. NSW DPI. 2012.

3. Bogema DR, Deutscher AT, Fell S, Collins D, Eamens G, Jenkins, C. Development and Validation of a Quantitative PCR Assay Using Multiplexed Hydrolysis Probes for Detection and Quantification of Theileria orientalis Isolates and Differentiation of Clinically Relevant Subtypes. J. Clin. Microbiol. 2015; 53: 941-950.

4. De Vos AJ. Theileria: Assess potential to develop a vaccine for Theileria orientalis infection. 2011. MLA report B.AHE.0076.

5. Eamens GJ, Bailey G, Gonsalves JR, Jenkins C. Distribution and temporal prevalence of Theileria orientalis major piroplasm surface protein types in eastern Australian cattle herds. Aust. Vet. J. 2013a; 8: 332-340. 
6. Eamens GJ, Gonsalves JR, Jenkins C, Collins D, Bailey G. Theileria orientalis MPSP types in Australian cattle herds associated with outbreaks of clinical disease and their association with clinical pathology findings. Vet. Parasitol. 2013b;191: 209-17.

7. Emery DL, DeBurgh S, Dinh THHH, Rolls P, Carter, P. Merozoites of Theileria orientalis buffeli reduce parasitosis following challenge by ticks infested with T.orientalis ikeda (Parasit. Vectors (sunmitted)

8. Gibson MJ. The effects of Theileria orientalis Ikeda on bull fertility and libido. 2017. PhD Thesis. Massey University. https://mro.massey.ac.nz/bitstream/handle/10179/12824/02_whole.pdf? sequence.

9. Hammer JF, Emery D, Bogema D, Jenkins C. Detection of Theileria orientalis genotypes in Haemaphysalis Iongicornis ticks from southern Australia. Parasit. Vectors 2015; 8, 229. https://parasitesandvectors.biomedcentral.com/articles/10.1186/s13071-015-0839-9.

10. Hammer JF, Bogema D, Jenkins C, Emery D. Mechanical transfer of Theileria orientalis: possible roles of biting arthropods, colostrum and husbandry practices in disease transmission. Parasit. Vectors. 2016; 9: 34-39.

11. Ishihara T. Bovine piroplasmosis in Japan. Natl. Inst. Anim. Hlth. Quart. 1962; 2: 29-31.

12. Izzo MM, Poe I, Horadagoda N, De Vos AJ, House J K. Haemolytic anaemia in cattle in NSW associated with Theileria infection. Aust. Vet. J. 2010; 88: 45-51.

13. Jenkins C, Micallef M, Alex S, Collins, Djordevic, SP, Bogema DR. Temporal dynamics and subpopulation analysis of Theileria orientalis genotypes in cattle. Infection, Genet. Evol. 2015; 32: 199-207.

14. Kamau J, de Vos AJ, Playford M, Salim B, Kinyanjui P, Sugimoto C. Emergence of new types of Theileria orientalis in Australian cattle and possible cause of theileriosis outbreaks. Parasit. Vectors 2011; 4: 22-31.

15. Lane J, Jubb T, Shephard R, Webb-Ware J, Fordyce G. MLA Final Report: Priority list of endemic diseases for the red meat industries. 2015; Meat and Livestock Australia, Sydney, Australia. http://www.wormboss.com.au/files/pages/worms/roundworms/the-cost-ofroundworms/B.AHE.0010_Final_Report.pdf.

16. Lawrence KE, Gedye K, Pomroy WE. A longitudinal study of the effect of Theileria orientalis Ikeda type infection on three New Zealand dairy farms naturally infected at pasture. Vet. Parasitol. 2019; 276, 108977.

17. McAllister MM. Successful vaccines for naturally occurring protozoal diseases of animals should guide human vaccine research. A review of protozoal vaccines and their designs. Parasitology 2014; 141: 624-640.

18. McFadden AMJ, Rawdon TG, Mayer J, Makin J, Morley CM. Clough R, Tham K, Mullner, Geysen, D. An outbreak of haemolytic anaemia associated with infection of Theileria orientalis in naïve cattle. N.Z. Vet. J. 2011; 59: 79-85.

19. Minami T, Ishihara T, Fujita J. Bovine theileriosis and its control in Japan. "Advances in the Control of Theileriosis". Proceedings of an International Conference held at the International Laboratory for 
Research on Animal Diseases in Nairobi, 9-13th February, 1981, pp 94-96.

20. Neal RA, Garnham PCC, Cohen S. Immunisation against protozoal parasites. Brit. Med. Bull.1969; 25: 194-210.

21. Onuma M, Kubota S, Kakud, T, Aako Y, Asada M, Kabeya M, Sugimoto, C. Control of Theileria sergenti infection by vaccination. Trop. Anim. Hlth. Prod. 1997; 29: 119S-123S.

https://doi.org/10.1007/BF02632949

22. Ota N, Mizuno D, Kuboki N, Igarashi I, Nakamura Y, Yamashin H, Hanzaike, T, Fujii K, Onoe S, Hata H, Kondo S, Matsui S, Koga M, Matsumoto K, Inokuma H, Yokoyama N. Epidemiological survey of Theileria orientalis infection in grazing cattle in the eastern part of Hokkaido, Japan. Jap.J. Vet. Med. Sci. 2009; 71: 937-944.

23. Park J, Lee S, Chae J, Kwon , Lee, J. Immunization of recombinant membrane protein in Theileria sergenti. Kor. J. Vet. Clin. Med. 1999; 16: 328-331.

24. Perera PK, Gasser R, Firestone, SM, Anderson GA, Malmo J, Davis G, Beggs .S. Jabbar A. Oriental theileriosis in dairy cows causes a significant milk production loss. Parasit Vectors. 2014; 7: $73-81$.

25. Reik RF. Epidemiology and transmission of Theileria sp of cattle in Australia. Aust. Vet. J. 1982; 59: 89-92.

26. Sivakumar T, Hayashida K, Sugimoto C, Yokoyama N. Evolution and genetic diversity of Theileria. Inf. Genet. Evol.2014; 27: 250-263.

27. Stewart NP, Standfast N.F, Baldock FC, Reid DJ, DeVos AJ. The distribution and prevalence of Theileria buffeli in cattle in Queensland. Aust. Vet. J. 1992; 69: 59-61.

28. Stewart N, de Vos AJ, McGregor W, Shiels I. The experimental transmission of Theileria buffeli of cattle in Australia by Haemaphysalis humerosa. Aust. Vet .J. 1987a; 64: 81-3.

29. Stewart NP, de Vos AJ, McGregor W, Shiels, I. Haemaphysalis humerosa, not H. longicornis, is the likely vector of Theileria buffeli in Australia. Aust. Vet. J. 1987b; 64: 280-282. doi:10.1111/j.17510813.1987.tb15960.x

30. Stewart, N.P., Uilenberg, G., de Vos, A.J. 1996. Review of Australian species of Theileria, with special reference to Theileria buffeli of cattle. Tropical Animal Health \& Production, 28:81-90. doi:10.1007/BF02250731.

31. Swilks, E., Fell, S.S., Hammer, J.F., Sales, N., Krebs, G.L., Jenkins, C. 2017. Transplacental transmission of Theileria orientalis occurs at a low rate in field-affected cattle: infection in utero does not appear to be a major cause of abortion. Parasit. Vectors 10, 227-236.

\section{Figures}




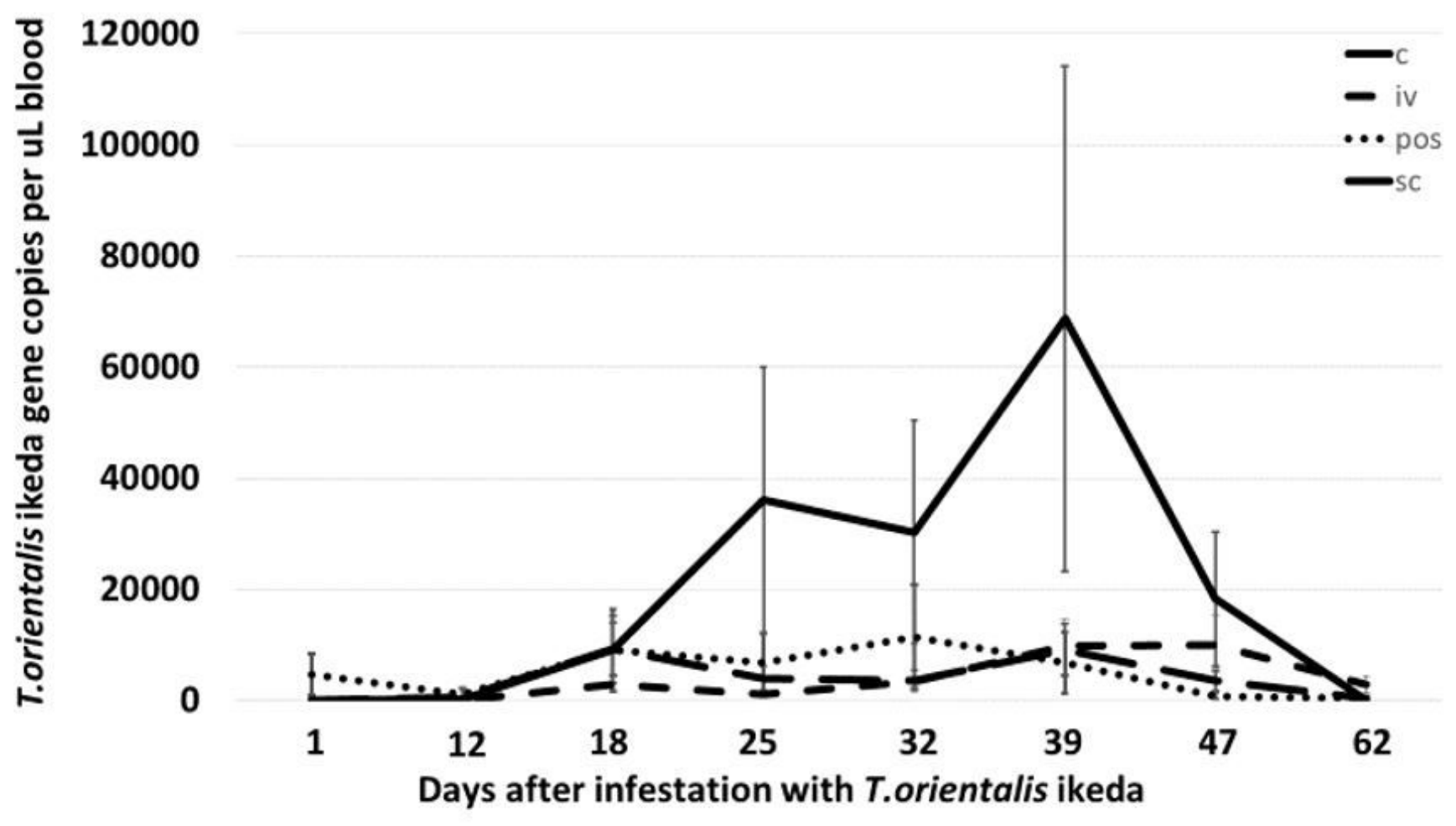

Figure 1

Group mean parasitoses for T.orientalis ikeda (GC/ per ul blood +/- SD) following infestation with 200 H.longicornis nymphs (see M\&M). Group comparisons include: uninfected controls (Gp 3; 3 calves, solid line); previously infected controls ( 2 calves, dotted line); groups of 5 calves immunised with T.orientalis buffeli either IV (Gp1; short dash) or SC (Gp2; long dash). 


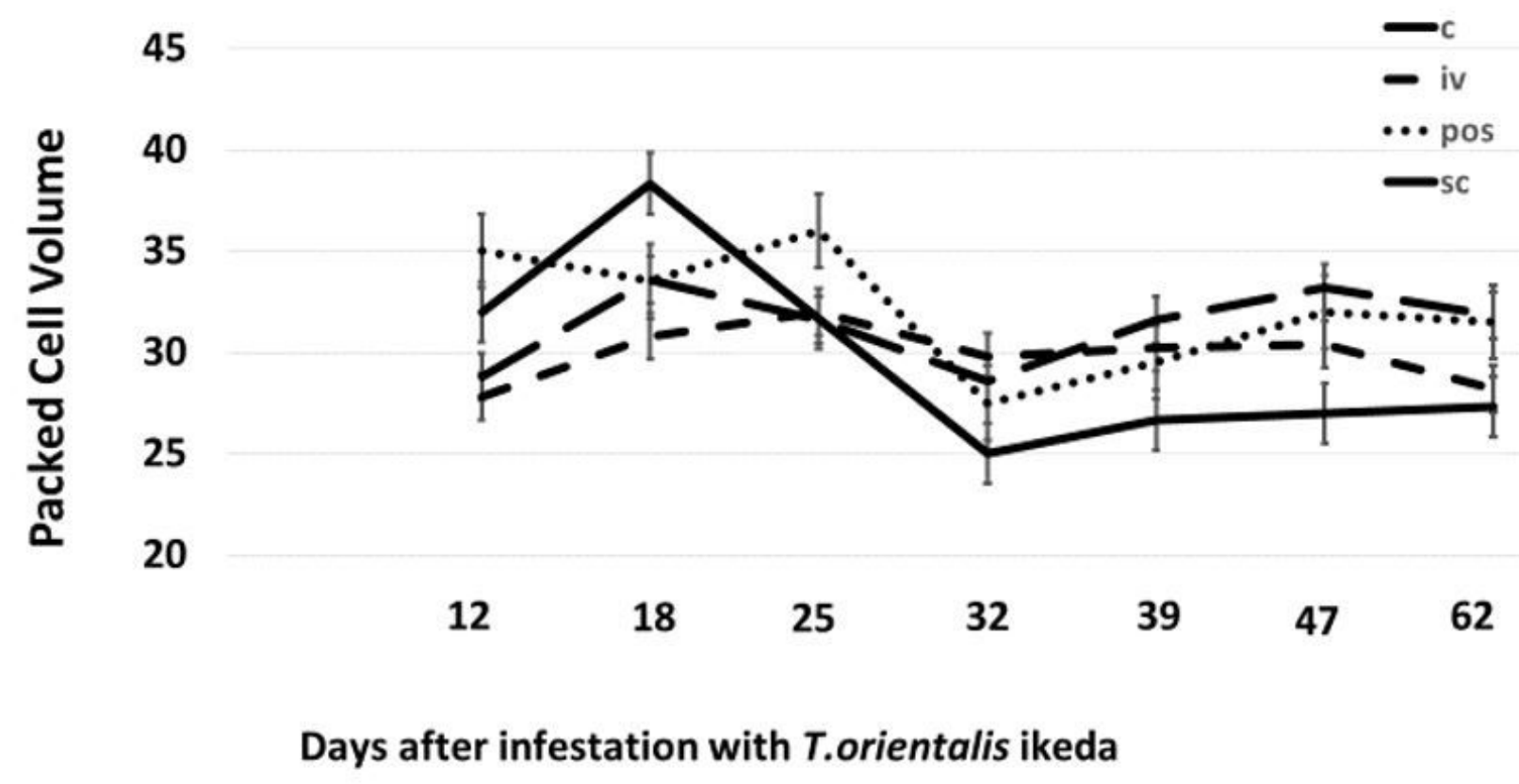

\section{Figure 2}

Group mean haematocrit (\% +/-SD) following infestation with 200 H.longicornis nymphs (see M\&M). Group comparisons include: uninfected controls, (Gp 3; 3 calves, solid line); previously infected controls ( 2 calves, dotted line); groups of 5 calves immunised with T.orientalis buffeli either IV (Gp1; short dashes) or SC (Gp2; long dash). 